\title{
Splanchnic and Peripheral Glucose and Lactate Metabolism during and after Prolonged Arm Exercise
}

\author{
Gunvor Ahlborg, John Wahren, and Philip Felig \\ Department of Clinical Physiology, Karolinska Institute, Södersjukhuset and Huddinge University Hospital, Stockholm, Sweden; and \\ Sandoz Pharmaceutical, Inc., East Hanover, New Jersey 07936
}

\begin{abstract}
Splanchnic and peripheral exchange of glucose and gluconeogenic substrates was examined in 12 healthy subjects during $2 \mathrm{~h}$ of arm or leg exercise on a bicycle ergometer and during a 40 -min postexercise recovery period. The work intensity corresponded to $30 \%$ of the maximal pulmonary oxygen uptake. The regional exchange of substrates was evaluated using catheter technique and indicator dilution methods for blood flow measurements.

Our findings indicate that prolonged arm exercise as compared with exercise with the legs results in a greater increase in heart rate (25-40\%) and a more marked reduction in splanchnic blood flow (10-30\%) as well as higher arterial concentrations of lactate, free fatty acids, and catecholamines. The respiratory exchange ratio was consistently higher with arm exercise. In addition, arm exercise results in a greater fractional extraction and utilization of glucose by exercising muscle as well as a greater hepatic gluconeogenesis from lactate and glycerol.

During recovery from prolonged arm exercise, leg muscle becomes an important site of lactate release to the splanchnic bed, despite a lack of net glucose uptake by the leg. Simultaneously, arm muscle shows an increase in glucose uptake in the absence of a net release of lactate. These coincident but discordant processes in the leg and arm during recovery suggest the occurrence of a redistribution of muscle glycogen from previously resting (leg) muscle to previously exercising (arm) muscle.
\end{abstract}

\section{Introduction}

Exercise is known to stimulate glucose utilization by muscle and at the same time increase hepatic glucose production and hepatic uptake of gluconeogenic precursors such as lactate, alanine, and glycerol $(1,2,3,4)$. The observations demonstrating these effects of exercise on glucose turnover and gluconeogenesis are virtually entirely based upon studies of leg rather than arm exercise. In previous studies arm exercise has been shown to result in greater increases in heart rate, blood pressure, and blood lactate concentration than those observed with leg exercise of the same intensity $(5,6)$. The effect of arm as compared with leg exercise on splanchnic exchange of glucose and gluconeogenic substrates and on the importance of glucose as a fuel for exercising muscle has not, however, been established. Such data are of particular interest since previous studies have suggested that sympathetic nervous system activity may be greater with arm exercise as compared with leg exercise $(5,6)$. Alterations in sympathetic

Address reprint requests to Dr. Wahren, Dept. of Clinical Physiology, Huddinge University Hospital, S-141 86 Stockholm, Sweden.

Received for publication 25 September 1985.

J. Clin. Invest.

(c) The American Society for Clinical Investigation, Inc.

$0021-9738 / 86 / 03 / 0690 / 10 \quad \$ 1.00$

Volume 77, March 1986, 690-699 nervous system activity and/or circulating catecholamines could be expected to influence such processes as glycogenolysis, gluconeogenesis, lipolysis, and muscle glucose utilization.

Also of interest is the metabolic response of relatively inactive muscle during and after prolonged exercise. In a recent study it was observed that during prolonged leg exercise and in the recovery period after leg exercise, the arm is a site of lactate release (7). However, in that study the quantitative significance of resting muscle as a source of lactate production could not be precisely determined since blood flow to the arm was not measured (7).

Consequently, we undertook this study to evaluate splanchnic and peripheral metabolism of glucose and gluconeogenic substrates during prolonged arm exercise, and to compare these changes with those observed during leg exercise of the same relative work intensity. We also examined substrate exchange across the resting leg during arm exercise and splanchnic and peripheral (arm and leg) metabolism in the recovery period after arm exercise.

\section{Methods}

Subjects. 12 healthy nonobese males were studied in the postabsorptive state after a 12-14-h overnight fast. The subjects were physically active and well trained but did not participate in competitive athletics. Data on age, height, weight, and maximal oxygen uptake are presented in Table I. For 2-3 d immediately before the study period the subjects were told to ingest meals consisting of $200-300 \mathrm{~g}$ of carbohydrate per day. All subjects were informed of the nature, purpose, and possible risks involved in the study before giving their voluntary consent to participate. The procedures employed in these studies have been reviewed and approved by the institutional ethical committees.

Procedure. The subjects were studied at rest, during $2 \mathrm{~h}$ of upright, continuous arm or leg exercise on a bicycle ergometer, and during a 40-min postexercise recovery period. Six subjects performed arm exercise, the other six performed leg exercise. The subjects were in the sitting position throughout the exercise. The axle of the ergometer during arm exercise was at the level of the shoulders. The workload during both arm and leg exercise was adjusted so that oxygen consumption corresponded to $\sim 30 \%$ of the maximal oxygen uptake $\left(\mathrm{V}_{2} \max \right)$ as determined by standard techniques (Table I). The workload for arm exercise was $25 \%$ lower than for leg exercise (Table I). Particular care was taken to limit leg activity as much as possible during arm exercise, by keeping the legs in a comfortable position with the knees bent at a $90^{\circ}$ angle.

A Cournand catheter (No. 8) was introduced percutaneously into a femoral vein and advanced to a right-sided hepatic vein under fluoroscopic control, and teflon catheters were inserted percutaneously into both femoral veins, a femoral artery, a deep forearm vein, and an axillary vein. During blood sampling from the deep forearm vein, hand circulation was blocked by a wrist cuff inflated to $230 \mathrm{mmHg}$.

Patency of the catheters was maintained by intermittent flushing with saline; the hepatic venous catheter was flushed with $1.5 \%$ sodium citrate solution, a total of $<0.3 \mathrm{~g}$ being administered over the course of the study. Indocyanine green dye was infused intraarterially (femoral artery) at a constant rate for estimation of leg (8) and hepatic blood flow (9). 
Table I. Age, Height, Weight, Maximal

Oxygen Uptake, and Work Load in Subjects

Performing Prolonged Arm or Leg Exercise

\begin{tabular}{llcc}
\hline & & Mean \pm SE & Range \\
\hline Age $(y r)$ & Arm ex & $27 \pm 1$ & $24-29$ \\
& Leg ex & $27 \pm 2$ & $19-31$ \\
Height $(\mathrm{cm})$ & Arm ex & $186 \pm 4$ & $171-198$ \\
& Leg ex & $181 \pm 3$ & $170-194$ \\
Weight $(k g)$ & Arm ex & $80 \pm 6$ & $61-100$ \\
& Leg ex & $74 \pm 4$ & $62-93$ \\
Maximal oxygen & Arm ex & $4.1 \pm 0.3$ & $3.3-4.8$ \\
uptake $(l i t e r / m i n)$ & Leg ex & $3.9 \pm 0.2$ & $3.3-4.8$ \\
Oxygen uptake & Arm ex & $31 \pm 1.2$ & $28-36$ \\
$\begin{array}{l}\text { during ex } \\
\text { (\% of } \text { max })\end{array}$ & Leg ex & $30 \pm 1$ & $28-32$ \\
Work load during & & & \\
$\quad$ ex $(W)$ & Arm ex & $60 \pm 4^{*}$ & $50-70$ \\
\hline
\end{tabular}

* Significantly different from the corresponding value during leg exercise $(P<0.01)$.

Ex, exercise.

Analyses. Glucose (10), lactate (11), glycerol (12), and alanine (13) were analyzed in whole blood by enzymatic techniques. Plasma insulin was determined by radioimmunoassay (14). Plasma catecholamines were determined by a radioenzymatic technique (15). Oxygen saturation was measured spectrophotometrically (16), and hemoglobin concentration was measured by the cyanmethemoglobin technique (17). The hematocrit was measured with a microcapillary hematocrit centrifuge and corrected for trapped plasma. Expired air was analyzed by the Scholander microtechnique (18).

Data in the text and tables are given as mean \pm SE. Standard statistical methods have been employed (19); the paired $t$ test was used in evaluating changes from the basal state, and the unpaired $t$ test was employed in comparing arm exercise with leg exercise.

\section{Results}

\section{Exercise}

Heart rate, pulmonary and splanchnic oxygen uptake, and blood flow (Table II). The pulmonary oxygen uptakes rose fivefold in the arm and leg exercise studies $(P<0.001$, Table II). However, as the absolute workload was $25 \%$ lower with arm exercise $(P$ $<0.01$, Table I), mechanical efficiency was also lower compared with leg exercise $(17.7 \pm 0.8 \%$ vs. $24.5 \pm 0.8 \%, P<0.001)$. Heart rate during arm exercise was $\sim 25-40 \%$ higher than during leg exercise (Table II). The respiratory exchange ratio was consistently higher with arm exercise as compared with leg exercise $(P<0.05$, Table II).

Estimated hepatic blood flow (EHBF) ${ }^{1}$ did not differ in the arm and leg exercise groups in the basal state but tended to be lower throughout the arm exercise, the difference being statistically significant at $120 \mathrm{~min}(P<0.05)$. Splanchnic oxygen

1. Abbreviations used in this paper: A-AV, arterial-axillary vein; A-DV, arterial-deep vein; A-V, arterial venous; EHBF, estimated hepatic blood flow. uptake showed a $20 \%$ increase at $90 \mathrm{~min}$ of arm exercise $(P$ $<0.05$ ) but did not differ significantly from the basal value at other times during exercise. Splanchnic oxygen uptake was similar during arm and leg exercise.

The arterial-venous (A-V) differences for oxygen at rest revealed a higher value for deep forearm muscle (arterial-deep vein, A-DV) than for the whole arm (arterial-axillary vein, A-AV, $P<0.05$ ). During arm exercise these A-V differences for oxygen increased, reaching values of the same magnitude.

Leg blood flow rose approximately twofold $(P<0.01)$ and leg oxygen uptake rose fourfold $(P<0.001)$ during the first 40 min of arm exercise, and remained essentially unchanged during the rest of the exercise period. During leg exercise these values were three to three and a half times higher $(P<0.001)$.

Arterial concentrations (Table III). Although within the normal range, the mean arterial glucose level was somewhat lower in the arm exercise group in the basal state $(P<0.05)$. During exercise arterial glucose remained unchanged from basal in the arm exercise study but fell progressively to values $\sim 0.5 \mathrm{mmol} /$ liter below baseline by $2 \mathrm{~h}$ in the leg exercise group $(P<0.05)$. As previously reported $(5,6)$, the rise in arterial lactate with arm exercise was significantly higher than with leg exercise $(P<0.001)$ and rose fivefold during the first $20 \mathrm{~min}$ of exercise, reaching values of $2.80 \pm 0.26 \mathrm{mmol} /$ liter $(P<0.001 \mathrm{vs}$. basal). From then on there was a small decline up to $90 \mathrm{~min}$ of arm exercise. In addition, free fatty acid (FFA) levels in response to arm exercise became $0.5 \mathrm{mmol} /$ liter $(45 \%)$ greater than during leg exercise $(P<0.05)$ despite similar basal values. The rise in arterial glycerol was $40-50 \%$ higher during arm exercise $(P<0.05)$.

Arterial insulin fell $\sim 25 \%$ during both arm and leg exercise $(P<0.01)$. Arterial norepinephrine levels during arm exercise were $65-95 \%$ above those during leg exercise $(P<0.01)$. Although mean arterial epinephrine levels were two- to threefold higher during arm as compared with leg exercise, large interindividual variations prevented the differences from reaching statistical significance.

Splanchnic substrate exchange (Table IV). Splanchnic glucose production rose $125 \%$ during the first $\mathbf{4 0} \mathrm{min}$ of arm exercise, reaching values $155 \%$ above baseline at $2 \mathrm{~h}(P<0.001)$, and did not differ from that during leg exercise. Thus, the cumulative splanchnic output of glucose during the 120-min arm exercise period was similar to that with leg exercise $(256 \pm 32$ vs. $236 \pm 40$ mmol).

Splanchnic uptake of lactate during arm exercise rose approximately fivefold to $1.67 \pm 0.06 \mathrm{mmol} / \mathrm{min}$ at $20 \mathrm{~min}$ of exercise $(P<0.01$ vs. basal). The uptake of lactate was 2.5 - to 3.5 fold greater than during leg exercise $(P<0.05-0.01)$. This increase in splanchnic lactate uptake with arm exercise was not only a consequence of the higher arterial lactate level. During arm exercise splanchnic fractional extraction of lactate was 25$80 \%$ higher than during leg exercise (Fig. $1, P<0.05$ at 90 and 120 min of exercise). Splanchnic uptake of glycerol did not differ between arm and leg exercise.

Leg substrate exchange (Table IV). During arm exercise, glucose uptake by the legs increased four- to fivefold above basal values. Glucose uptake by the leg during leg exercise was, however, seven- to ninefold greater than during arm exercise. The fractional extraction of glucose by the leg (arterial-femoral venous difference/arterial glucose) at 90 and $120 \mathrm{~min}$ of leg exercise was $12 \%$, which is lower than the fractional extraction of glucose by the arm during arm exercise (Fig. 2; see also below). 


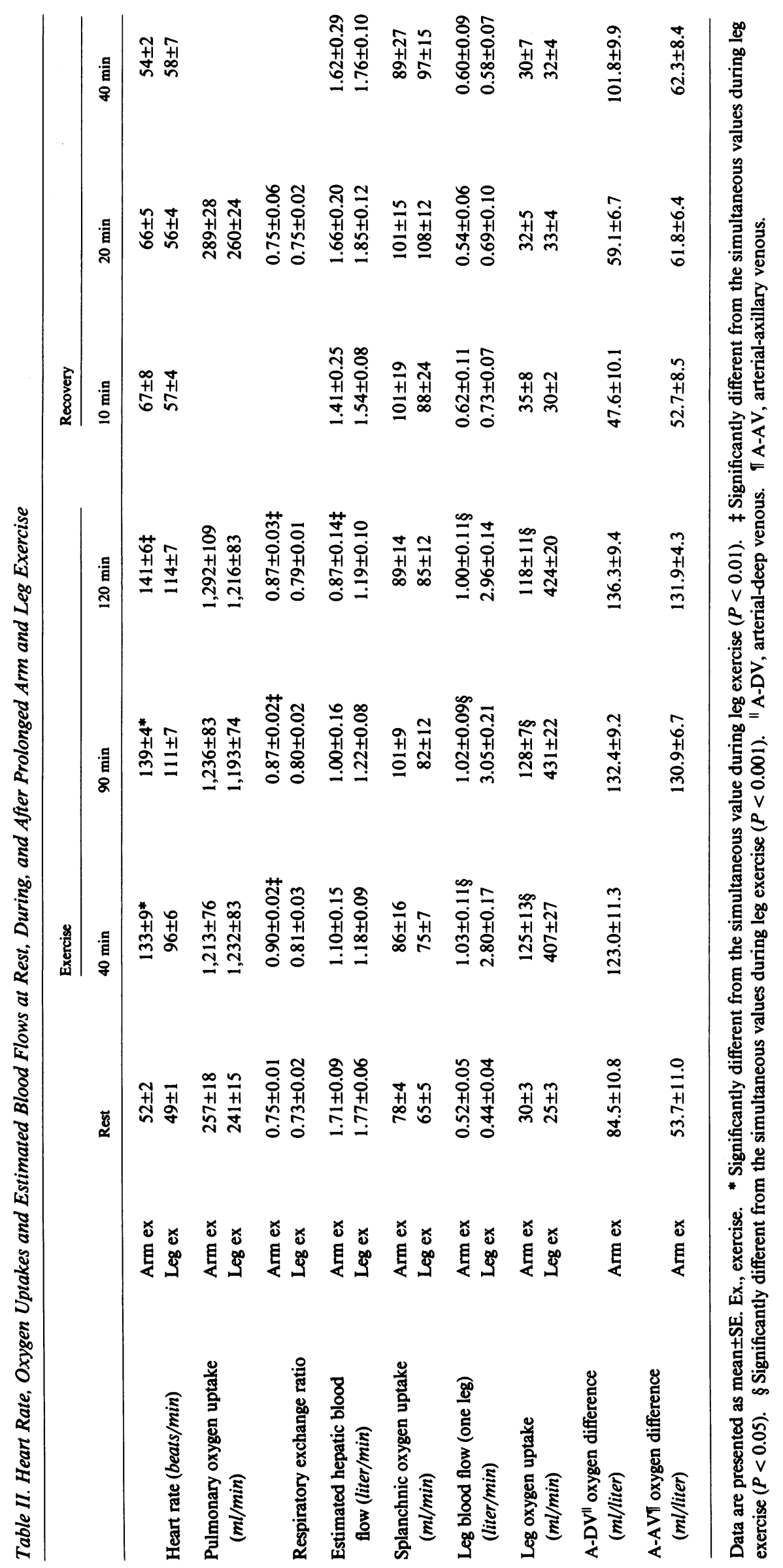




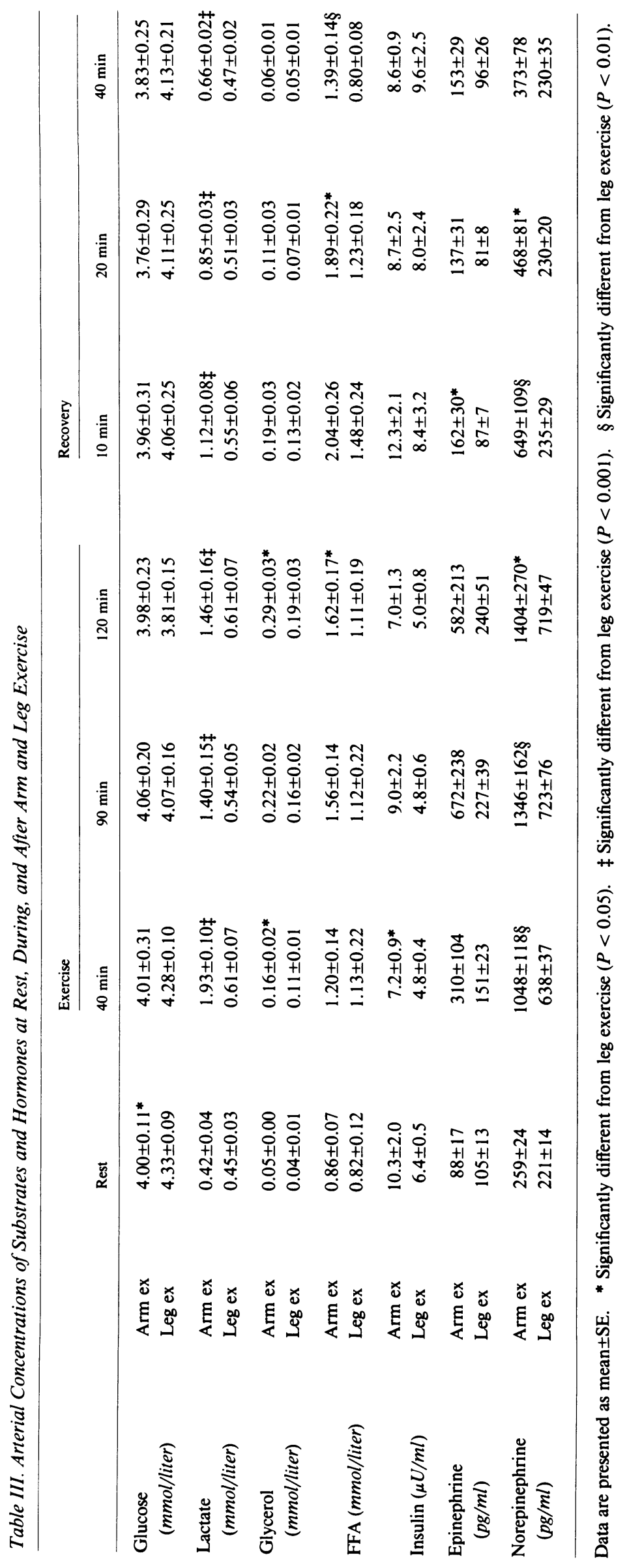




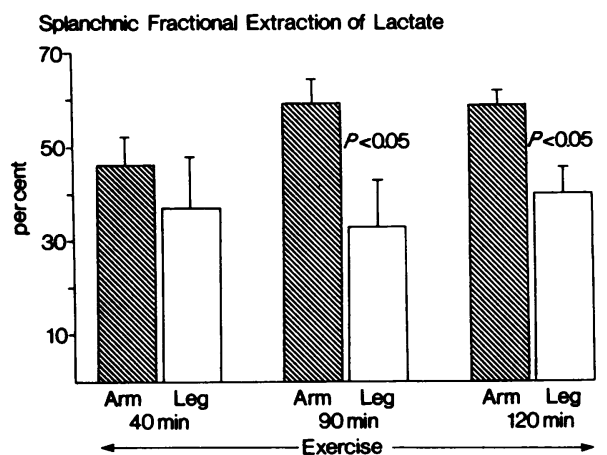

Figure 1. Splanchnic fractional extraction of lactate during arm and leg exercise. Fractional extraction calculated by dividing the arterialhepatic venous difference for lactate by the arterial lactate concentration.

There was a net release of lactate by the legs at rest. This was reversed to a net uptake of lactate at $20 \mathrm{~min}$ of arm exercise, amounting to $0.54 \pm 0.13 \mathrm{mmol} / \mathrm{min}(P<0.01$ vs. basal); an uptake of lactate by the leg was still noticed at $40 \mathrm{~min}$ of arm exercise (Table IV, $P<0.01$ ). In contrast, at $40 \mathrm{~min}$ of leg exercise there was lactate production by the legs. No significant net lactate exchange across the leg was observed at 90 or $120 \mathrm{~min}$ of arm or leg exercise.

There was a similar small net glycerol output by the legs during arm or leg exercise, except at $\mathbf{4 0}$ min of exercise, when glycerol output was higher during arm compared with leg exercise $(P<0.001)$.

Arm substrate exchange (Table V). During and after arm exercise arterial-venous differences for substrates were determined across the whole arm (A-AV differences), as well as across the deep tissues of the forearm (A-DV differences). The A-AV glucose difference rose five- to sixfold above basal during arm exercise $(P<0.01)$. The fractional extraction of glucose by the whole arm (A-AV/arterial glucose) after 90-120 min of arm exercise was $17-20 \%$, thus more than 1.5 -fold higher than that by leg during leg exercise $(P<0.05-0.001$, Fig. 2$)$.

The A-AV difference for lactate, which was negative in the basal state and at $90 \mathrm{~min}$ of arm exercise, was not significantly different from zero at $120 \mathrm{~min}$ of arm exercise. The A-AV differences for glycerol did not differ from zero during arm exercise.

With respect to the A-DV differences, the values for glucose remained unchanged during arm exercise. The A-DV differences for lactate revealed a net release in the basal state, which became more negative at $40 \mathrm{~min}$ of arm exercise $(P<0.05)$. Thereafter, there was a decline to values that at 90 and 120 min were not significantly different from zero. The A-DV differences for glycerol did not differ from zero in the basal state or during exercise.

\section{Recovery}

Heart rate, pulmonary and splanchnic oxygen uptake, and blood flow (Table II). Heart rate fell back rapidly, but remained slightly above basal for the first $20 \mathrm{~min}$ of recovery $(P<0.05)$. Pulmonary oxygen uptake, respiratory exchange ratio, EHBF, leg blood flow, leg oxygen uptake, and A-V oxygen differences across the arm and forearm all returned to basal values within $10 \mathrm{~min}$. There were no differences in heart rate, pulmonary oxygen uptake, or respiratory exchange ratio, EHBF, leg blood flow, or leg oxygen uptake in the recovery period after arm compared with leg ex- ercise, although leg blood flow remained above basal at 10 and $20 \mathrm{~min}$ after leg exercise $(P<0.05-0.01)$.

Arterial concentrations (Table III). Arterial lactate values fell during recovery, but remained $60 \%$ above basal at $40 \mathrm{~min}$ after arm exercise $(P<0.001)$. As compared with the leg exercise study, arterial lactate levels were still 40-100\% $(P<0.001)$ higher after arm compared with after leg exercise. Arterial glycerol and FFA levels were also $20-60 \%$ above basal at $20-40 \mathrm{~min}$ after arm exercise $(P<0.05)$. There was no difference in arterial glycerol levels in the recovery period after arm or leg exercise. In contrast, arterial FFA levels were $40-75 \%$ higher $(P<0.05-$ 0.01 ) following arm exercise as compared with leg exercise.

Plasma insulin had returned to basal values $10 \mathrm{~min}$ after arm and leg exercise. Plasma epinephrine and norepinephrine declined rapidly during recovery, but were still elevated at 10$20 \mathrm{~min}$ after arm exercise $(P<0.05)$. Plasma epinephrine levels were $60-85 \%$ higher after arm exercise compared with leg exercise $(P<0.05,10 \mathrm{~min}$ after exercise). Plasma norepinephrine levels were approximately two- to threefold higher up to $20 \mathrm{~min}$ after arm vs. leg exercise $(P<0.05-0.01)$.

Splanchnic substrate exchange (Table IV). Splanchnic glucose exchange returned to basal values within $10 \mathrm{~min}$ after arm exercise, with no difference compared with leg exercise. Splanchnic uptake of lactate remained two- to threefold greater than basal rates at 10 and $20 \mathrm{~min}$ after arm exercise $(P<0.05-0.0025)$. A small drop in lactate uptake was observed between 20 and 40 min of recovery but the value at 40 min was still $40 \%$ above basal $(P<0.05)$. Splanchnic lactate uptake was $45-85 \%$ higher up to $20 \mathrm{~min}$ of recovery after arm exercise as compared with leg exercise $(P<0.05)$. Splanchnic glycerol uptake remained two- to fourfold above basal for the initial $20 \mathrm{~min}$ of recovery $(P<0.05)$ and did not differ from the values after leg exercise.

Leg substrate exchange (Table IV). Leg uptake of glucose during recovery from arm exercise fell to values not significantly different from basal within $10 \mathrm{~min}$. Compared with recovery after leg exercise, glucose uptake by the legs was lower after arm exercise $(P<0.05-0.001)$. Lactate output from the leg after exercise increased to values that were six- to sevenfold greater than in the basal state $(P<0.01)$. This increased output of lactate from the leg persisted throughout the 40 -min recovery period (Table IV). Leg lactate output was one- to threefold higher after arm exercise compared with leg exercise $(P<0.05-0.001)$. There was no difference in leg exchange of glycerol after arm or leg exercise.

Arm substrate exchange (Table V). The A-AV difference for glucose during recovery increased to values 10 - to 14 -fold the basal rate $(P<0.005)$. In contrast, the A-AV lactate difference was not significantly different from zero throughout the recovery period. The A-AV glycerol differences returned to basal values after exercise.

The A-DV difference for glucose increased to values twofold the basal rate throughout recovery $(P<0.05-0.01)$. The A-DV lactate difference was more negative during recovery than in the basal state, the most pronounced change being observed at $\mathbf{4 0}$ min of recovery $(P<0.05)$. The A-DV glycerol difference also became more negative during recovery compared with the basal value $(P<0.05-0.01)$.

\section{Discussion}

The current findings demonstrate that at comparable levels of oxygen consumption ( $30-31 \%$ of $\dot{\mathrm{VO}}_{2}$ max), arm exercise results 


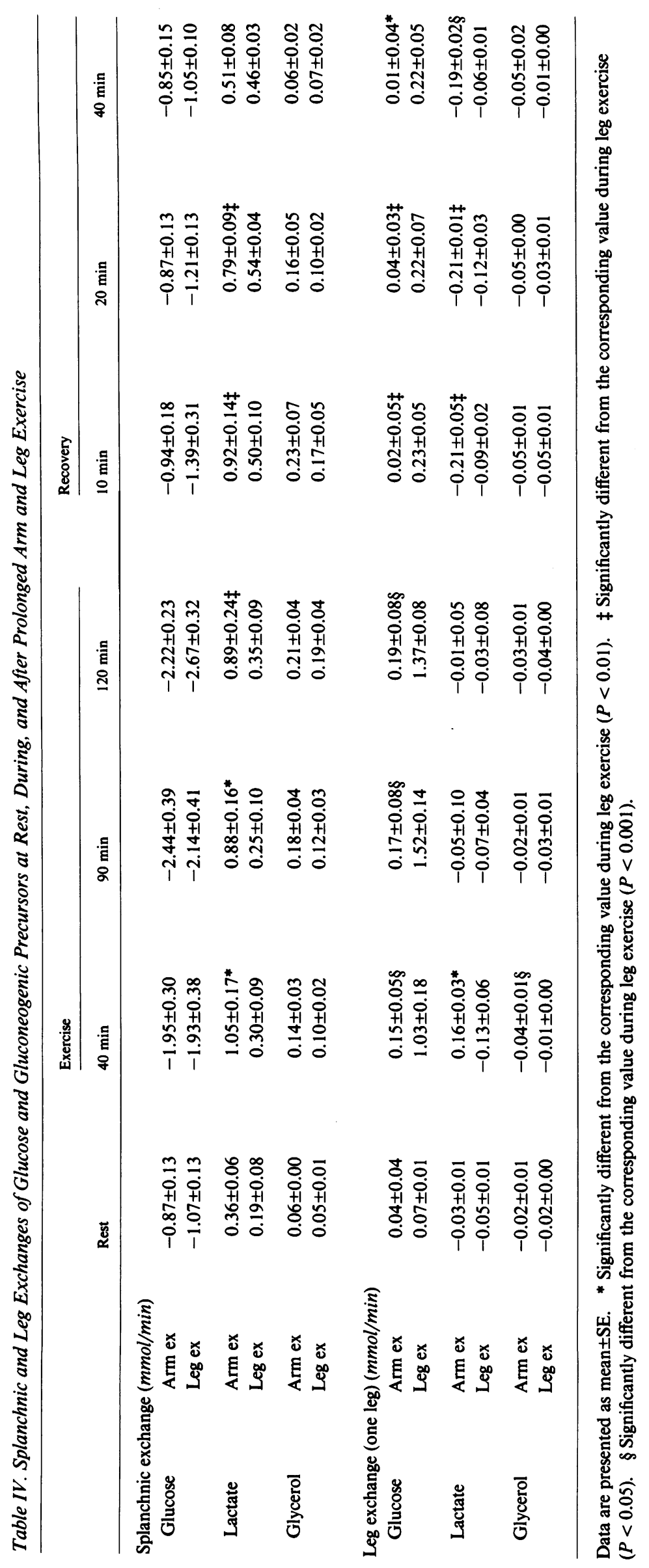




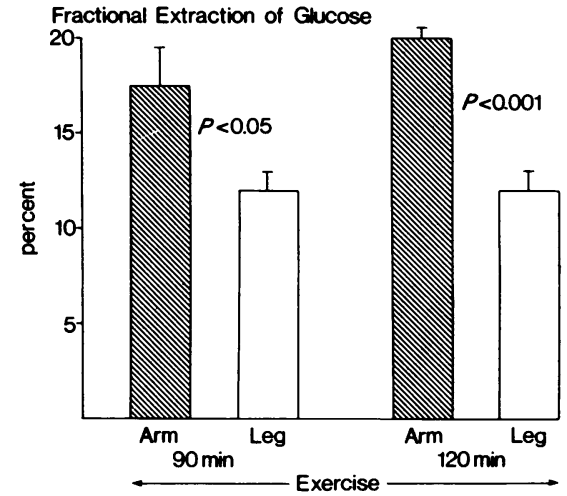

Figure 2. Fractional extraction of glucose by the exercising limb during arm or leg exercise. Fractional extraction calculated by dividing the arterial-axillary venous glucose difference (for arm exercise) or the arterial-femoral venous glucose difference (for leg exercise) by the arterial glucose concentration.

in metabolic as well as hemodynamic changes that differ from those observed with leg exercise. Although pulmonary oxygen consumption was virtually identical in the arm and leg exercise studies, the absolute workload (in watts) and consequently the whole body mechanical efficiency were reduced when exercise was performed with the arms as compared with the legs. These reductions may be the result of differences in the efficiency of oxygen utilization within the contracting muscles or, more likely, may reflect a greater increase in oxygen consumption by nonexercising parts of the body during arm exercise (20). In addition, the higher arterial catecholamine levels during arm exercise may have stimulated the oxidative metabolism.

In accord with earlier studies $(5,6)$, arm exercise resulted in a greater increase in heart rate than observed with leg exercise. In addition, the arterial norepinephrine levels became as much as $95 \%$ higher compared with leg exercise. Arm exercise also tended to result in a more marked fall in estimated hepatic blood flow. The present findings thus indicate that with respect to higher norepinephrine levels, reduction of splanchnic blood flow as well as acceleration of heart rate, the hemodynamic effects of arm exercise are comparable to those induced by leg exercise of considerably greater intensity.

Particularly striking were the differences in the metabolic response to arm exercise as compared with leg exercise as reflected by glucose, lactate, and FFA metabolism. In arm as well as leg exercise, increases were observed in splanchnic glucose production and peripheral glucose utilization. However, arm exercise had a greater stimulatory effect on both aspects of glucose turnover. Thus, although the absolute work performed was lower $(P<0.01)$ during arm exercise, the level of splanchnic glucose output during the 2-h exercise period was similar to that observed with leg exercise (Table IV). Furthermore, this relative increase in glucose output in relation to the absolute work load was associated with a $60-100 \%$ rise in splanchnic uptake of gluconeogenic precursors, particularly lactate. As a consequence, splanchnic uptake of gluconeogenic precursors (lactate and glycerol) during exercise could (if completely converted to glucose) account for $22-31 \%$ of splanchnic glucose output during arm exercise but only $9-10 \%$ during leg exercise $(P<0.05-0.01$, Table IV). Based on splanchnic glucose output minus uptake of gluconeogenic precursors, cumulated splanchnic glycogenolysis for the 2-h exercise period was estimated to $185 \pm 20$ and $212 \pm 33$ mmol with arm and leg exercise, respectively. These findings suggest that arm exercise may constitute a relatively greater stimulus than leg exercise not only for hepatic gluconeogenesis but also for glycogenolysis.

In addition to the relative increase in glucose production, the data also provide evidence of a greater contribution of glucose to fuel utilization by the exercising arm as compared with the exercising leg. Since blood flow to the arm was not determined, glucose uptake by the arm could not be quantitated precisely. However, from the measurements of A-V differences for glucose and oxygen, one can assess the relative contribution of bloodborne glucose to total oxidative metabolism. Since the arm exercise involved extensive contraction of the biceps and triceps muscles and far less contraction of the forearm muscles, A-AV differences were considered to be more representative of overall arm metabolism than the A-DV differences across the forearm. This is further supported by the higher A-AV differences for glucose as compared with the forearm deep vein (Table V, $P$

Table V. Arterial-Venous Differences for Glucose and Gluconeogenetic Substrates across the Whole Arm and the Deep Tissues of the Forearm at Rest, During, and After Prolonged Arm Exercise

\begin{tabular}{|c|c|c|c|c|c|c|c|}
\hline & \multirow[b]{2}{*}{ Rest } & \multicolumn{3}{|l|}{ Arm exercise } & \multicolumn{3}{|l|}{ Recovery } \\
\hline & & $40 \mathrm{~min}$ & $90 \mathrm{~min}$ & $120 \min$ & $10 \min$ & $20 \mathrm{~min}$ & $40 \mathrm{~min}$ \\
\hline \multicolumn{8}{|c|}{$\mathrm{A}-\mathrm{AV}^{*}(\mathrm{mmol} /$ liter $)$} \\
\hline Glucose & $0.11 \pm 0.07$ & & $0.57 \pm 0.10$ & $0.67 \pm 0.13$ & $1.13 \pm 0.19$ & $1.52 \pm 0.28$ & $1.21 \pm 0.32$ \\
\hline Lactate & $-0.06 \pm 0.02$ & & $-0.23 \pm 0.09$ & $-0.09 \pm 0.14$ & $0.00 \pm 0.05$ & $-0.10 \pm 0.07$ & $-0.11 \pm 0.09$ \\
\hline Glycerol & $-0.04 \pm 0.02$ & & $-0.02 \pm 0.02$ & $0.01 \pm 0.02$ & $-0.05 \pm 0.01$ & $-0.06 \pm 0.01$ & $-0.03 \pm 0.01$ \\
\hline \multicolumn{8}{|c|}{ A-DV $\ddagger$ (mmol/liter) } \\
\hline Glucose & $0.33 \pm 0.08$ & $0.32 \pm 0.05$ & $0.44 \pm 0.08$ & $0.37 \pm 0.07$ & $0.77 \pm 0.15$ & $0.65 \pm 0.11$ & $0.72 \pm 0.14$ \\
\hline Lactate & $-0.12 \pm 0.02$ & $-0.37 \pm 0.14$ & $-0.22 \pm 0.12$ & $-0.03 \pm 0.08$ & $-0.19 \pm 0.12$ & $-0.29 \pm 0.09$ & $-0.31 \pm 0.08$ \\
\hline Glycerol & $-0.01 \pm 0.01$ & $0.00 \pm 0.00$ & $-0.01 \pm 0.01$ & $0.00 \pm 0.01$ & $-0.06 \pm 0.01$ & $-0.08 \pm 0.01$ & $-0.05 \pm 0.02$ \\
\hline
\end{tabular}

Data are presented as mean $\pm \mathrm{SE}$. * A-AV, arterial-axillary venous difference. $\ddagger \mathrm{A}-\mathrm{DV}$, arterial-deep venous difference. 


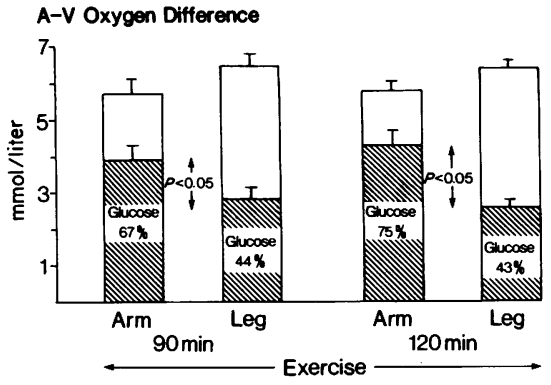

Figure 3. The contribution of glucose to total oxidative metabolism by the exercising limb during arm or leg exercise. The height of the bar represents the A-V oxygen difference across the arm (arterial-axillary venous difference) or leg (arterial-femoral venous difference) during arm and leg exercise, respectively. The cross-hatched areas indicate the A-V differences for glucose expressed in oxygen equivalents. The percent values represent the proportion of total oxygen uptake that can be accounted for by oxidation of glucose.

$<0.025$ at $120 \mathrm{~min}$ ). As shown in Fig. 3, net glucose uptake by the exercising arm could account for $67-75 \%$ of total arm oxidative metabolism. In contrast, during leg exercise, glucose uptake by the exercising leg could account for only $43-44 \%$ of total oxidative metabolism. This evidence of greater dependence on glucose utilization during arm vs. leg exercise is supported by the data on the respiratory exchange ratio $(R)$, with higher values during arm exercise (Table II).

The greater dependence on glucose utilization in arm exercise as compared with leg exercise could not be ascribed to an increase in glucose delivery. Thus, the arterial blood glucose concentration was not higher during arm exercise. Furthermore, fractional extraction of glucose by the exercising limb was higher with arm as compared with leg exercise (Fig. 2). These findings thus suggest that arm exercise has a relatively greater stimulatory effect on peripheral glucose utilization than is observed with leg exercise of comparable intensity. Note that the greater dependence on blood glucose utilization during arm exercise occurred in the face of arterial FFA levels that were higher than those observed with leg exercise.

With respect to lactate metabolism, an exaggerated rise in blood lactate in response to arm exercise has previously been reported $(5,6)$. The hyperlactatemia of arm exercise could conceivably be a consequence of enhanced lactate production, decreased lactate utilization, particularly by the liver (4), or a combination of the two. The present data indicate that splanchnic uptake of lactate is markedly increased in arm exercise as compared with leg exercise. This rise in lactate uptake was primarily a consequence of increased lactate delivery to the splanchnic bed. Also, fractional extraction of lactate by the splanchnic tissues was greater with arm exercise as compared with leg exercise (Fig. 1). Arm exercise thus exerts a greater stimulatory effect on hepatic uptake of lactate than is observed with leg exercise.

Concerning lactate production, the presence of increased arterial lactate levels in association with an augmented lactate uptake by the splanchnic tissues, indicates that peripheral (extrasplanchnic) production of lactate is stimulated to a greater extent with arm exercise as compared with leg exercise. With respect to the site(s) of lactate production, a significant net release of lactate by the arm (as reflected by either A-AV or A-DV differences) was not demonstrable beyond $90 \mathrm{~min}$ of arm exercise.
These data are in keeping with previous observations in which the exercising leg failed to demonstrate a net release of lactate beyond $90 \mathrm{~min}$ (7). In the latter study, we observed that arm muscle becomes an important source of lactate release during prolonged leg exercise performed at $58 \%$ of $\mathrm{VO}_{2} \max (7)$. It was therefore of interest in the present study to examine lactate exchange across the leg during prolonged arm exercise. In contrast to the leg exercise study (7), we did not detect any net release of lactate from the nonexercising limb (the legs) during arm exercise. These differences may relate to the greater intensity of the exercise in the leg exercise study (7), since lactate production was observed by the resting legs in the recovery period. The extrasplanchnic site of lactate production during arm exercise beyond 90 min thus remains undefined. Nevertheless, the current data underscore the conclusion that tissues other than the exercising extremity may be an important source of lactate production during prolonged (>90 $\mathrm{min}$ ) exercise (7).

With respect to the mechanism(s) whereby arm exercise induces differing metabolic and hemodynamic responses from those observed with leg exercise, we have already mentioned the alterations in neurohumoral responses. In addition, differences in the intensity of the exercise performed by individual muscle groups, and/or differences in fiber composition or enzyme activity of arm vs. leg muscle, may be of importance. Since a greater rise in blood pressure as well as heart rate has previously been noted with arm exercise, a greater increase in activity of the sympathetic nervous system has been postulated to accompany arm exercise $(5,6)$. Note that the magnitude of the rise in plasma norepinephrine as well as epinephrine observed with arm exercise in the present study is comparable to that observed with leg exercise of far greater intensity (7). An increase in sympathetic nervous system activity and/or circulating catecholamines would be expected to result in an enhancement of splanchnic glycogenolysis and gluconeogenesis, an increase in peripheral lactate production (due to accelerated muscle glycogenolysis), an increased production of FFA, and (via alpha adrenergic receptors) a decreased splanchnic blood flow (21). It would not, however, account for the greater dependence on glucose as an oxidative fuel. A possible contributory factor to these findings regarding glucose metabolism during arm exercise might be the somewhat higher insulin levels found during arm exercise compared with leg exercise (Table III). The differing responses to arm exercise as compared with leg exercise (including an enhanced sympathetic-adrenergic response) may also be a direct consequence of the smaller muscle mass in the arms compared with the legs, necessitating a greater intensity of contraction by individual muscle groups. Note, however, that doubling the intensity of leg exercise (from 30 to $58 \% \mathrm{VO}_{2} \max$ ) fails to increase the proportion of leg oxygen consumption attributable to glucose uptake. Furthermore, threefold increases in the intensity of leg exercise (from 65 to $196 \mathrm{~W}$ ) fail to enhance the fractional extraction of glucose by the exercising leg (22). Thus, it is unlikely that increased intensity of contraction by individual muscle fibers or groups is the major factor responsible for the enhanced extraction and dependence on blood-borne glucose observed with arm exercise compared with leg exercise.

The possibility must also be considered that in view of the weight-bearing and locomotor activities to which the legs but not the arms are normally exposed, there may be differences in fiber composition and/or enzyme activity of arm muscle as compared with leg muscle. The proportion of slow-twitch and 
fast-twitch fibers, however, has not been shown to differ in arm (e.g., deltoideus, biceps) and leg muscles (e.g., vastus lateralis, gastrocnemius) (23). On the other hand, the content of oxidative enzymes influenced by training (e.g., succinate dehydrogenase) tends to be lower in arm muscle than in leg muscle (24). Regardless of the mechanism(s) involved, the present findings clearly demonstrate that the metabolic response to arm exercise differs from that observed with leg exercise with respect to the production and utilization of glucose as well as lactate and circulating levels of FFA.

The current study also provided an opportunity to examine the metabolic changes that occur during recovery from prolonged arm exercise. Although blood flow to the arm (or forearm) was not measured, it is unlikely that blood flow during recovery was lower than observed in the basal state. The data on A-AV differences thus indicate that glucose uptake by the arm during the recovery period was at least 10 - to 15 -fold greater than in the basal state. These findings are in keeping with earlier observations of increased glucose uptake during recovery by the previously exercising leg $(7,25)$. The demonstration that this enhanced glucose uptake occurs in association with plasma insulin levels that are not elevated above basal levels (Table II), supports the conclusion that the postexercise recovery period is characterized by increased insulin sensitivity in the previously exercising muscle $(7,26,27)$.

Of particular note were the findings with respect to lactate exchange during the recovery period. In a previous study, resting forearm muscle was shown to release lactate during and after prolonged leg exercise (7). This lactate release was greater than the simultaneous glucose uptake, indicating glycogenolysis within the muscle as a lactate source (7). The quantitative significance of lactate release by previously resting muscle could not, however, be determined in that study since blood flow to the arms was not measured (7). The current findings show that during recovery from arm exercise, leg release of lactate was six- to sevenfold greater than in the basal state. Moreover, the combined output of lactate from the two legs could account for $40-75 \%$ of the splanchnic lactate uptake during recovery (Table IV). Although the data on oxygen and glucose uptake indicate that the legs are not totally at rest during arm exercise, the extent of contraction in the legs is far less than in the arms. The current data thus indicate that during recovery from arm exercise, previously "resting" (relatively inactive) muscle in the legs is the major source of lactate delivery to the splanchnic bed. Forearm muscle (as reflected by A-DV) differences also constitute a source of lactate release in the recovery period.

It is of interest to compare the data on glucose uptake and lactate release in arm, forearm, and leg muscle during recovery from arm exercise. In the recovery period, the arm (as reflected by $\mathrm{A}-\mathrm{AV}$ differences) has a markedly increased glucose uptake (as compared with basal) but no consistent net release of lactate, which suggests that glycogen repletion is occurring in the previously exercising arm muscle. In the forearm (as reflected by A-DV differences), glucose uptake as well as lactate release are stimulated, which suggests that in muscle that was less vigorously exercised, glycogen stores may remain unchanged in the recovery period. In marked contrast, in the leg, glucose uptake is unchanged from basal, while lactate output is increased six- to sevenfold, indicating stimulation of glycogenolysis in previously "resting" (relatively inactive) muscle. These changes in peripheral glucose and lactate metabolism occur in a setting of markedly

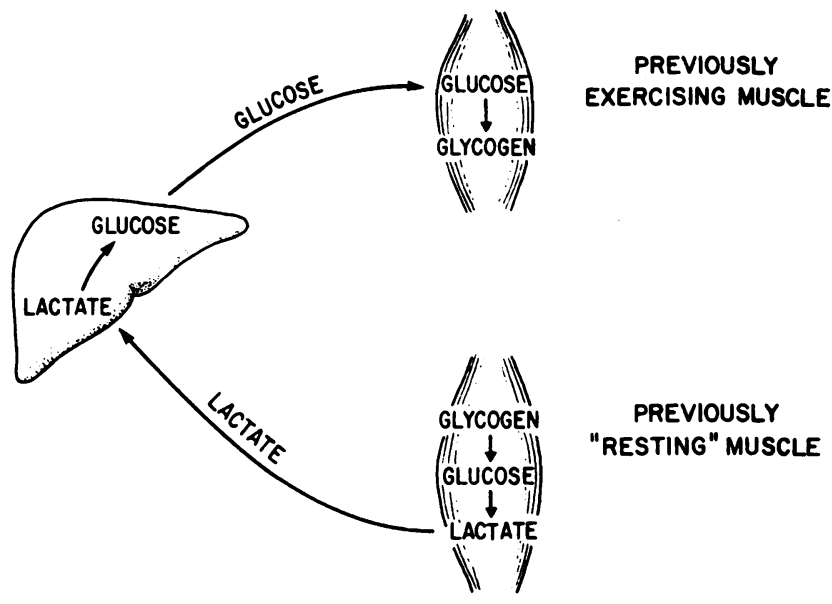

Figure 4. Postulated interaction of previously exercising muscle and previously "resting" (relatively inactive) muscle via the Cori cycle during recovery from prolonged arm exercise. During recovery, glucose uptake by previously exercising muscle (i.e., the arm) is increased above basal levels in the absence of any significant net release of lactate, which suggests muscle glycogen repletion. In contrast, in the previously "resting" muscle (i.e., the leg), lactate output is markedly increased above basal levels, accounting for $40-75 \%$ of splanchnic lactate uptake. This occurs in the absence of a net uptake of glucose, suggesting breakdown of glycogen in previously resting muscle. The net effect of these simultaneous but discordant processes in the arm and leg, in association with an increase in splanchnic lactate uptake, is a redistribution of muscle glycogen from previously resting to previously exercising muscle.

augmented uptake of lactate by the splanchnic bed during the recovery period. These observations thus provide strong support for the conclusion that the Cori cycle (conversion by the liver of glucose-derived lactate to glucose) provides a mechanism for the redistribution of muscle glycogen during recovery from previously resting muscle to previously exercising muscle (Fig. 4) (7). In keeping with this formulation, an increase in glycogen content of previously exercising muscle has been observed during recovery from prolonged exercise, even in the absence of food ingestion (28). Biopsy studies examining the glycogen content of previously resting muscle during recovery from prolonged exercise have not been reported.

\section{Acknowledgment}

This study was supported in part by grants from the Swedish Medical Research Council (3108) and the Research Council of the Swedish Sports Federation (27/82).

\section{References}

1. Ahlborg, G., P. Felig, L. Hagenfeldt, R. Hendler, and J. Wahren. 1974. Substrate turnover during prolonged exercise in man. Splanchnic and leg metabolism of glucose, free fatty acids, and amino acids. J. Clin. Invest. 53:1080-1090.

2. Felig, P., and J. Wahren. 1976. Fuel homeostasis in exercise. $N$. Engl. J. Med. 293:1078-1083.

3. Vranic, M., and M. Berger. 1979. Exercise and diabetes mellitus. Diabetes. 28:147-163.

4. Rowell, L. B., G. L. Brengelmann, J. R. Blackman, R. D. Twiss, and F. Kusumi. 1966. Splanchnic removal of lactate and pyruvate during prolonged exercise in man. J. Appl. Physiol. 21:1773-1783. 
5. Bevegård, S., U. Freyschuss, and T. Strandell. 1966. Circulatory adaptation to arm and leg exercise in supine and sitting position. J. Appl. Physiol. 21(1):37-46.

6. Freyschuss, U. 1975. Comparison between arm and leg exercise in women and men. Scand. J. Clin. Lab. Invest. 35:795-800.

7. Ahlborg, G., and P. Felig. 1982. Lactate and glucose exchange across the forearm, legs, and splanchnic bed during and after prolonged leg exercise. J. Clin. Invest. 69:45-54.

8. Jorfeldt, L., and J. Wahren. 1971. Leg blood flow during exercise in man. Clin. Sci. (Lond.). 41:459-473.

9. Rowell, L. B., J. R. Blackmon, and R. Bruce. 1964. Indocyanine green clearance and estimated hepatic blood flow during mild to maximal exercise in upright man. J. Clin. Invest. 43:1677-1690.

10. Huggett, A. S. G., and D. A. Nixon. 1957. Use of glucose oxidase, peroxidase and o-dianisidine in determination of blood and urinary glucose. Lancet. ii:368-370.

11. Wahren, J. 1966. Quantitative aspects of blood flow and oxygen uptake in the human forearm during rhythmic exercise. Acta Physiol. Scand. 67(Suppl. 269):1-92.

12. Wieland, O. 1962. Glycerin. In Methoden der enzymatischen Analyse. H. U. Bergmeyer, editor. Verlag-Chemie, Weinheim, Bergstrasse, West Germany. 211-214.

13. Karl, I. E., A. S. Pagliara, and D. M. Kipnis. 1972. A microfluorometric enzymatic assay for the determination of alanine and pyruvate in plasma and tissues. J. Lab. Clin. Med. 80:434-444.

14. Rosselin, G., R. Assan, A. Yalow, and S. A. Berson. 1966. Separation of antibodybound and unbound peptide hormones labelled with iodine- 131 by talcum powder and precipitated silica. Nature (Lond.). 212:355-357.

15. Cryer, P. E. 1976. Isotope-derivative measurements of plasma norepinephrine and epinephrine in man. Diabetes. 25:1071-1082.

16. Drabkin, D. L. 1950. Measurement of $\mathrm{O}_{2}$-saturation of blood by direct spectrophotometric determination. Methods Med. Res. 2:159-162.

17. Drabkin, D. L., and J. H. Austin. 1935. Spectrophotometric studies. II. Preparations from washed blood cells; nitric oxide hemoglobin and sulfhemoglobin. J. Biol. Chem. 112:51-65.

18. Scholander, P. F. 1947. Analyzer for accurate estimation of respiratory gases in one-half cubic centimeter samples. J. Biol. Chem. 167: 235-240.

19. Snedecor, G. W., and W. G. Cochran. 1967. Statistical methods, 6th ed. Iowa State University Press, Ames, IA.

20. Ahlborg, G., L. Hagenfeldt, and J. Wahren. 1975. Substrate utilization by the inactive leg during one-leg or arm exercise. J. Appl. Physiol. 35:718-723.

21. Weiner, N. 1980. Norepinephrine, epinephrine and the sympathometric amines. In The Pharmacological Basis of Therapeutics. 6th ed. A. G. Gilman, L. S. Goodman, and A. Gilman, editors. Macmillan Publishing Co., New York. 138-176.

22. Wahren, J., P. Felig, G. Ahlborg, and L. Jorfeldt. 1971. Glucose metabolism during leg exercise in man. J. Clin. Invest. 50:2715-2725.

23. Saltin, B., J. Henriksson, E. Nygaard, P. Andersen, and E. Jansson. 1977. Fiber types and metabolic potentials of skeletal muscle in sedentary man and endurance runners. Ann. N.Y. Acad. Sci. 301:3-29.

24. Gollnick, P. D., R. B. Armstrong, C. W. Saubert IV, K. Piehl, and B. Saltin. 1972. Enzyme activity and fiber composition in skeletal muscle of untrained and trained men. J. Appl. Physiol. 33:312-319.

25. Wahren, J., P. Felig, R. Hendler, and G. Ahlborg. 1973. Glucose and amino acid metabolism during recovery after exercise. J. Appl. Physiol. 34:838-845.

26. Richter, R., A. Gainello, M. Goodman, and N. Ruderman. 1982. Muscle glycogen metabolism following exercise in the rat. Increased sensitivity to insulin. J. Clin. Invest. 69:785-793.

27. Calles, J., J. Cunningham, L. Nelson, N. Brown, E. Nadel, R. S. Sherwin, and P. Felig. 1983. Glucose turnover during recovery from intensive exercise. Diabetes. 32:734-738.

28. Maehlum, S., and L. Hermansen. 1978. Muscle glycogen concentration during recovery after prolonged severe exercise in fasting subjects. Scand. J. Clin. Lab. Invest. 38:557-560. 